Research Article

\title{
Vehicle-Borne Transient Electromagnetic Numerical Characteristic Parameter of Water-Bearing Body behind Tunnel Linings
}

\author{
Wangping Qian (iD, ${ }^{1,2}$ Taiyue Qi (iD, ${ }^{1,2}$ Xiao Liang, ${ }^{1,2}$ Shaojie Qin, ${ }^{1,2}$ Zongyang Li, ${ }^{1,2}$ \\ and $\operatorname{Yan} \mathrm{Li}^{1,2}$ \\ ${ }^{1}$ MOE Key Laboratory of Transportation Tunnel Engineering, Southwest Jiaotong University, Chengdu 610031, China \\ ${ }^{2}$ School of Civil Engineering, Southwest Jiaotong University, Chengdu 610031, China \\ Correspondence should be addressed to Taiyue Qi; qitaiyue58@126.com
}

Received 3 September 2019; Revised 19 January 2020; Accepted 17 February 2020; Published 16 March 2020

Academic Editor: Pietro Bia

Copyright (c) 2020 Wangping Qian et al. This is an open access article distributed under the Creative Commons Attribution License, which permits unrestricted use, distribution, and reproduction in any medium, provided the original work is properly cited.

\begin{abstract}
The water-bearing body (WBB) behind tunnel linings has been some of the main causes of damage in operational tunnels. The WBB directly affects the serviceability state of tunnel linings; thus, determining a method to detect this problem is a widely studied issue regarding tunnel maintenance. In this paper, a vehicle-borne transient electromagnetic method (VBTEM) is put forward for the first time to detect WBB behind tunnel linings, and the aim is to fully investigate the transient electromagnetic response and numerical characteristics of the WBB behind tunnel linings. Firstly, the transient electromagnetic response curves of the WBB and surrounding rock are obtained and compared in detail by using the finite element method. Then, taking the distance, thickness, radius, and resistivity of the WBB as variable parameters, the parametric sensitivity rule of the response curve of the WBB is analyzed. Finally, a dimensionless response curve is proposed, a mathematical extraction equation is established, and seven numerical characteristic parameters are proposed and extracted. Based on seven numerical characteristic parameters, the technical parameters of the VBTEM equipment are put forward. The results illustrate that the transient electromagnetic response of the WBB is obviously different from that of surrounding rock. The seven numerical characteristic parameters of the dimensionless curve of the WBB can represent the entire response curve of the WBB. The results reveal that the first time gate of the VBTEM equipment needs to be less than $0.05 \mu$ s for short-distance problem, and the time span needs to reach four time spans at least. The research results can provide valuable technical references for the application of VBTEM.
\end{abstract}

\section{Introduction}

Water is a serious problem in tunnel maintenance $[1,2]$. Water-bearing body (WBB) has been proven to exist behind the tunnel linings of many operational tunnels [3, 4]. Moreover, the WBB has also been validated as a source of damage to many tunnel linings, such as water leakage, lining creaks, and concrete deterioration $[5,6]$, and the WBB can be identified as the origin of damage to many tunnel linings in operational tunnels to some extent. If the tunnel waterproofing fails, flowing water will accumulate behind the tunnel lining, which will seriously affect tunnel structure safety. Thus, the detection of the hidden WBB behind tunnel linings has become highly necessary for tunnel engineers. To solve this problem, the vehicle-borne transient electromagnetic method (VBTEM) is put forward to detect the hidden WBB behind tunnel linings.

The VBTEM is based on improved detection by the transient electromagnetic method (TEM) in many engineering applications, such as geological advance prediction of tunnels, underground gas storage, and metal mineral exploration [7-14]. The VBTEM has the common characteristics of transient electromagnetic in other fields, but also has its differences, which means that some issues need to be discussed before applying VBTEM technology into operational tunnels. Firstly, ground TEM and aviation TEM 
usually work on half-space problems $[7,9]$, but like mine TEM and tunnel TEM, VBTEM often considers full-space problems. Furthermore, there may be enough planes or spaces to layout the transmitter and receiver coils in other TEM applications $[8,11,13]$, whereas VBTEM only has a limited space depended on the size and form of the tunnel cross section, and thus, the size of the coils is constrained and only a limited number of measurement points can be measured. Finally, the detection distance of the low-resistivity target of VBTEM is different from the traditional TEM. For example, Fitterman and Stewart [7] studied the transient electromagnetic sounding for groundwater exploration at approximately $1000 \mathrm{~m}$ with a $100 \times 100 \mathrm{~m}^{2}$ transmitter loop, Hördt [8] adopted an innovative instrumentation with a $40 \times 40 \mathrm{~m}^{2}$ transmitter loop and found an unknown and unexpected buried valley complex at $300 \mathrm{~m}$, Li et al. [14] predicted and detected water-filled caves ahead of the tunnel excavation face at $30 \mathrm{~m}$ with a $2 \times 2 \mathrm{~m}^{2}$ transmitter loop. However, the WBB behind tunnel linings of VBTEM is too close. Based on the abovementioned differences, the detection of the WBB behind tunnel linings is a full-space and short-distance problem.

In operational tunnels, the WBB behind tunnel linings within the range of $2 \mathrm{~m}$ to $5 \mathrm{~m}$ is an urgent concern for tunnel engineers, which means that detection distance of the low-resistivity target of VBTEM is different from other TEM and the corresponding electromagnetic response between them are also different. The time range requirement of ground TEM and tunnel TEM range from microsecond level to millisecond level $[9,13,14]$, while the time range requirement of VBTEM is required to be at least microsecond level or lower, so the transmitter type and coil parameters of VBTEM need to be reselected and redesigned, respectively. Because of the lower level of time range, the early transient electromagnetic signals need to be paid more attention, which means the technical parameters of the VBTEM equipment should be proposed. To early-time and shortdistance TEM problem, the response curve law of the WBB behind tunnel linings is also worth of further study. Meanwhile, unlike ground TEM and tunnel TEM, the scale of the WBB cannot be very large, and it exists on a certain scale behind the tunnel lining; thus, whether its scale can be effectively detected remains to be further investigated. Due to the limitation of tunnel cross section, only a limited number of measurement points can be measured in operational tunnels, just like tunnel TEM $[14,15]$, so the singlepoint electromagnetic signal technology also needs to be referenced in VBTEM. Similarly, in the case of a single measurement point and short-distance, detecting the WBB behind tunnel linings in operational tunnels needs to be profoundly investigated, and physical characteristics of the WBB behind tunnel linings also needs to be further described.

To solve the problems of the WBB above, taking a typical tunnel detection model as the research object, a threedimensional electromagnetic numerical simulation model is established by the finite element method. The transient electromagnetic response curves of the uniform full-space surrounding rock and $\mathrm{WBB}$ are investigated, and the difference between the two curves is comprehensively analyzed. Through the parametric sensitivity analysis of the $\mathrm{WBB}$, the transient electromagnetic response rule of the WBB at different parameters is fully studied. Based on the research idea of normalization, the dimensionless electromagnetic response curves of the $\mathrm{WBB}$ are presented to clearly highlight the change in the WBB. The numerical characteristic parameters at the single point electromagnetic signal are fully introduced from a quantitative perspective. Finally, the mathematical extraction equation is proposed, and seven numerical characteristic parameters are extracted. Aimed at the short-distance of the WBB, the technical parameters of VBTEM equipment are put forward. This research provides valuable technical references for the selection of transmitter and design of coils of VBTEM.

\section{Analysis Principle and Calculation Method}

2.1. Introduction to Tunnel Detection System. TEM technology has been widely used for the detection of different media $[7,10,14]$. In operational tunnels, the normal surrounding rock and WBB have very different electrical resistivity; thus, the transient electromagnetic technology has the potentiality and ability to detect the normal surrounding rock and WBB behind tunnel linings, and it can even investigate the development scale of the WBB. In this paper, the VBTEM is first proposed to detect the WBB behind tunnel linings. The VBTEM measurements in operational tunnels mainly involve the VBTEM coil and the vehicle equipment, where the VBTEM coil contains the transmitter coil and the receiver coil. A detailed layout of the VBTEM detection system is shown in Figure 1. The function of the transmitter coil is to form the primary magnetic field in the surrounding rock space, and the function of the receiver coil is to receive the secondary magnetic field caused by the eddy currents in the surrounding rock behind tunnel linings.

The realization process of VBTEM detection is to install the transient electromagnetic equipment and coils on the vehicle equipment. The vehicle equipment provides a working platform for the coils and a moving function for subsequent rapid detection, so the VBTEM system can detect and move at the same time, which means that the system can acquire the transient electromagnetic induction signals when the system is moving. During the operation of vehicle equipment, a certain frequency and power of transient electromagnetic pulse signal is launched to the tunnel structure and surrounding rock, the special receiver coil receives the transient electromagnetic induction signals of the tunnel structure and surrounding rock. When the vehicle equipment passes through the whole tunnel, the transient electromagnetic induction signal of the whole tunnel can be obtained. Through postprocessing of transient electromagnetic induction signals, the disease characteristics of the tunnel structure can be identified, the spatial location of the corresponding internal disease can be obtained, and the distribution map of the disease in the whole tunnel structure can be determined.

The VBTEM system can sense a single transient to capture three-dimensional (3D) spatial information about 


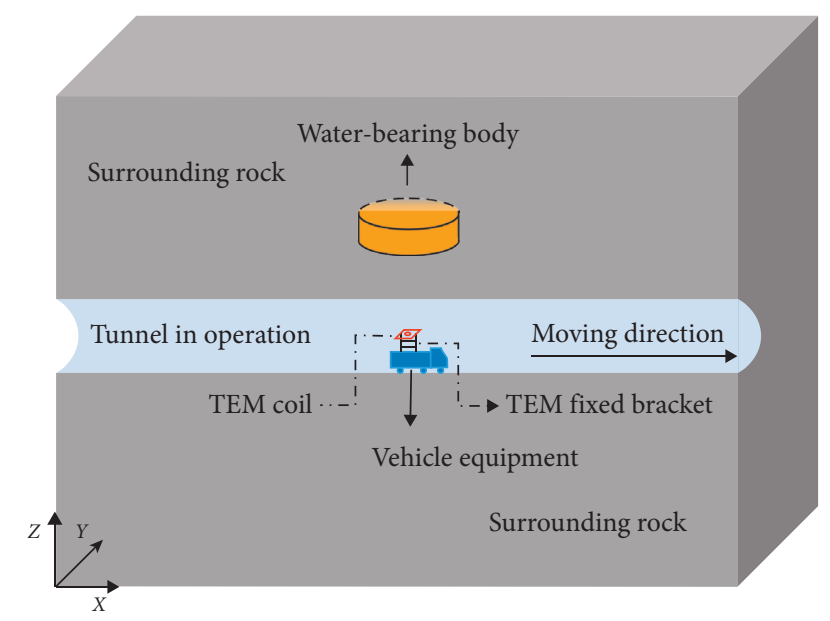

FIGURE 1: Sketch of the VBTEM detection system of the WBB behind tunnel linings.

surrounding rock. TEM technology has been widely used in the aviation, marine, well drilling, and tunnel excavation field for decades, but the VBTEM system discussed in this paper is an important attempt in operational tunnels.

2.2. Transient Theory of Electromagnetic Fields. The basic principle of the VBTEM measurement system in operational tunnels is shown in Figure 2. The current of the transmitter coil is suddenly switched off at time $t_{0}$. This abrupt change in the primary magnetic field induces an impulse of electromotive force in the surrounding rock, and this electromotive force will cause eddy currents to flow in the surrounding rock. The receiver coil measures the rate of change in the secondary magnetic field generated by the diffusing eddy currents in the time range $\tau \in\left[t_{1}, t_{2}\right]$. Although the basic principle of the VBTEM is same as that of other applications of TEM, the first time gate $t_{1}$ of the VBTEM needs to be more earlier due to the short-distance of the WBB. Therefore, it is an early transient electromagnetic problem, which is an important difference between the VBTEM and others TEM. Meanwhile, the turn-off time $t_{\text {off }}$ based on the existing equipment of TEM needs to be shortened due to the redesigned-coil of VBTEM and the first time gate.

To facilitate the analysis of transient electromagnetic fields in space, the following assumptions are made:

(1) The surrounding rock is a perfect linear isotropic medium. The medium is source-free and lacks additional polarization and magnetization media. The correction of the complexity of the properties of surrounding rock mass is not considered in this paper.

(2) The effect of the displacement current is ignored, and the propagation effects in the time range of interest are neglected.

(3) The current of the transmitter coil adopts the ideal step wave in the process of the theoretical analysis of the electromagnetic field. In general, only one-step wave excitation is usually discussed to simplify the problem.

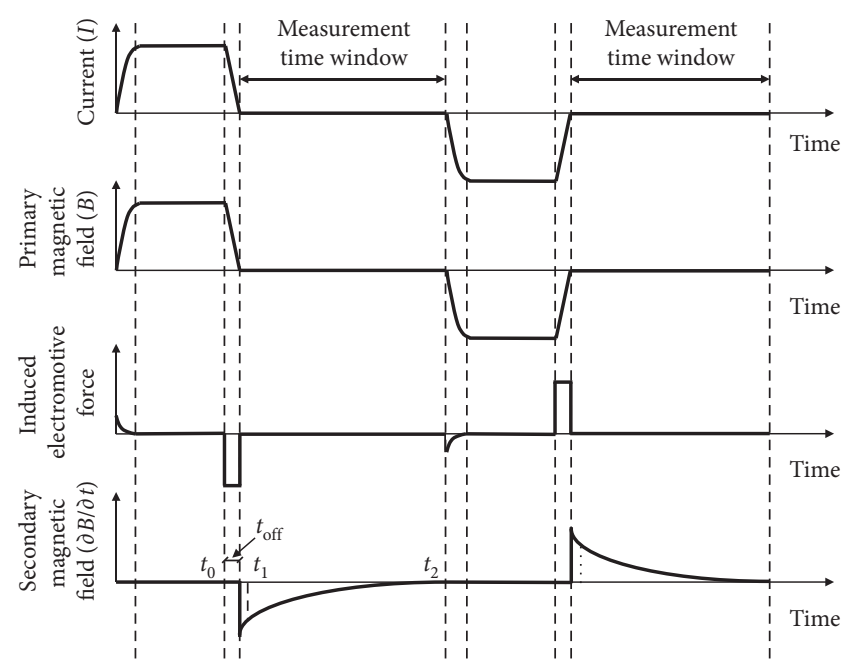

FIgURE 2: Basic principle of the VBTEM measurement system in operational tunnels.

(4) The interference sources, such as vehicles, steel frames, and rock bolts, are not considered, and only the effect of the WBB is considered.

Based on the above assumptions, the governing equations for VBTEM diffusion can be illustrated by simplifying Maxwell's equations as follows:

$$
\begin{aligned}
\nabla \times E & =-\frac{\partial}{\partial t} B, \\
\nabla \times H-\sigma \cdot E & =0,
\end{aligned}
$$

where $E$ is the electric field intensity $(\mathrm{V} / \mathrm{m}), H$ is the magnetic field intensity $(\mathrm{A} / \mathrm{m}), B$ is the magnetic flux density $\left(\mathrm{Wb} / \mathrm{m}^{2}\right), \sigma$ is the electric conductivity tensor for an anisotropic formation $(\mathrm{S} / \mathrm{m})$, and $t$ is the time of the electromagnetic field (s).

Assuming that the research problem of this paper considers free space, $\varepsilon=\varepsilon_{0}=8.854 \times 10^{-12} \mathrm{~F} / \mathrm{m}$ and $\mu=\mu_{0}=4 \pi \times 10^{-7} \mathrm{H} / \mathrm{m}$, where $\varepsilon_{0}$ and $\mu_{0}$ are the vacuum dielectric constant $(\mathrm{F} / \mathrm{m})$ and vacuum magnetic permeability $(\mathrm{H} / \mathrm{m})$, respectively.

Introducing the vector magnetic potential $A$ defined as $B=\nabla \times A$, we can rewrite formulas (1) and (2) as

$$
\sigma \cdot \frac{\partial}{\partial t} A+\nabla \times H=0
$$

Generally, the transmitter coil is fed with an ideal negative step current and its form is shown in formula (8).

$$
I(t)= \begin{cases}I_{0}, & t<0 \\ 0, & t \geq 0\end{cases}
$$

where $I(t)$ presents the negative step wave, $I_{0}$ represents the current in the transmitter coil, and $t$ denotes the time of the transient electromagnetic system.

The amplitude of the induced voltage caused by the secondary magnetic field in the receiver coil is correlated to the surrounding rock behind tunnel linings. Based on 
Faraday's electromagnetic induction law, the induced voltage of the receiver coil is shown in formula (5):

$$
V(t)=-N \frac{\partial \phi}{\partial t}=-N S \frac{\partial B}{\partial t}
$$

where $V(t)$ is the induced voltage in the receiver coil, $\phi$ is the magnetic flux in the receiver coil, $N$ is the number of turns in the receiver coil, and $S$ is the area of the receiver coil.

2.3. Numerical Model. The secondary electromagnetic field signals in a homogenous isotropic media have been investigated via theoretical analysis and numerical simulation $[10,14,16]$, and the results of these methods are consistent when the error is allowed; thus, the numerical simulation method is also adopted in this paper. The COMSOL Multiphysics (Version 5.4) is selected as the simulation software based on the principle of finite element, and the AC/DC module is specially designed to solve the simulation problem of electromagnetic field. Meanwhile, a two-dimensional rotationally symmetric model can simplify the threedimensional space model under the condition of space symmetry, and it has been proven to have good accuracy and efficiency. As shown in Figure 1, the three-dimensional space is a completely symmetric model when the topographic changes in surrounding rocks and the existence of tunnels are not considered; thus, the two-dimensional rotationally symmetric model is adopted to simulate the three-dimensional space model. In the numerical calculation, formula (3) is selected as the governing equation. To focus on the transient electromagnetic response of the $\mathrm{WBB}$, the steel frames in the lining concrete, the air in the tunnel trunk, the vehicle equipment, and the topography of surrounding rock are not considered in this paper. Meanwhile, the space problem of VBTEM is not constant because of the topography of surrounding rock, and the full-space problem chosen in this paper is an ideal and fundamental condition.

Similar to most coil configurations, the central loop configuration of the VBTEM coil is also adopted in operational tunnels. To facilitate the establishment of the finite element model, the transmitter coil is simplified as a circular coil based on the principle of coil area equivalence, and the WBB is assumed to be a flat cylinder. Any area outside the tunnel cross section can be the location of the $\mathrm{WBB}$, but we simplify the position of the WBB to the normal direction of the transmitter coil, which is the most basic case of different locations. The detailed layout and dimensions of the VBTEM coil and WBB are shown in Figure 3, where $I$ and $l$ are the current and diameter of the transmitting coil, respectively, $\rho, D$, and $R$ represent the resistivity, thickness, and radius of the WBB, respectively, and $L$ denotes the distance between the transmitter coil and the WBB.

The boundary condition imposed on all external boundaries is magnetic insulation, which means that $n \times A$ is equal to 0 . The outer boundaries should be placed far enough, so they do not affect the solution in the region of interest. To simulate infinite space and prevent reflection of

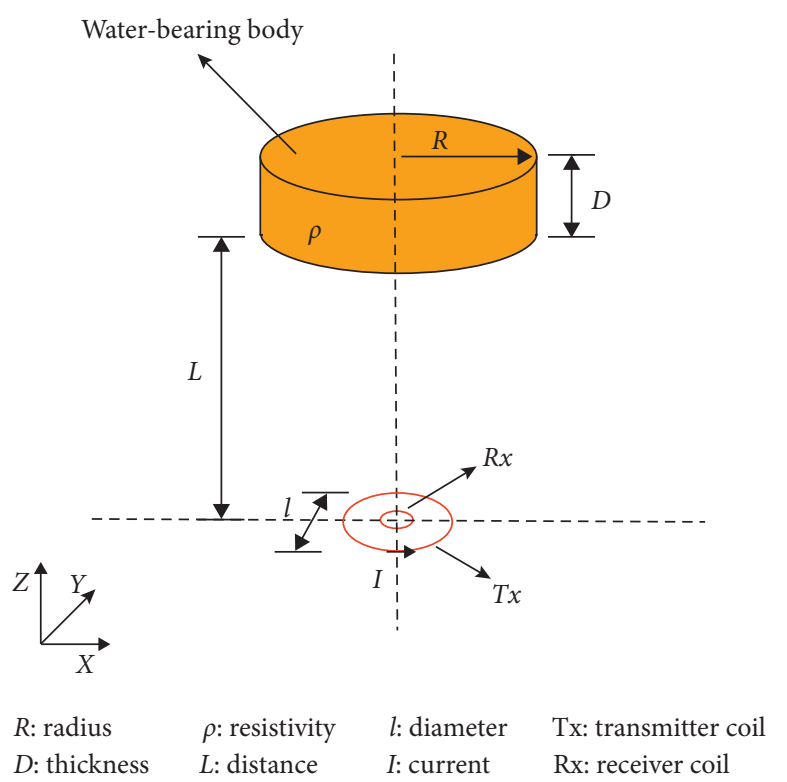

Figure 3: Layout and dimensions of the VBTEM coil and WBB.

the electromagnetic wave at the boundary, an infinite element boundary with a thickness of $20 \mathrm{~m}$ is set for the external surrounding rock. In the simulation model, the radius of the surrounding rock is $400 \mathrm{~m}$, and the height of the surrounding rock is $800 \mathrm{~m}$. The resistivity of the surrounding rock is not static, and its range is from $100 \Omega \cdot \mathrm{m}$ to $8000 \Omega \cdot \mathrm{m}$. For the sake of convenience, the resistivity of the surrounding rock is set as $1000 \Omega \cdot \mathrm{m}$ based on the literature [7-10]. For the dimensions of the WBB, the distance between the abnormal body and the transmitter coil is $4 \mathrm{~m}$, the radius of the $\mathrm{WBB}$ is $4 \mathrm{~m}$, the thickness of the $\mathrm{WBB}$ is $0.3 \mathrm{~m}$, and the resistivity of the $\mathrm{WBB}$ is $0.5 \Omega \cdot \mathrm{m}$. The finite element model is shown in Figure 4 . Because of the huge size difference between the WBB and surrounding rock, it is hard to illustrate all the details in the same figure, so a local magnification area to highlight the WBB and coil is shown in Figure 4. Because the model is much larger than the research object, the grids of the $\mathrm{WBB}$ and transmitter coil should be reasonably refined to achieve a high-accuracy calculation. A reasonably fine grid is defined for the regions of interest and for those with high contrasts of electromagnetic properties, including the coil and $\mathrm{WBB}$, the maximum grid of the cell is one-tenth of the corresponding object, and the local grid is shown in Figure 4. A reasonably coarse mesh is kept away from these regions for fast computation.

During transient electromagnetic geophysical exploration, the transmitter coil is square, and the square coil is simplified to a circular coil for the sake of model calculation. The transmitter coil is a line current loop, and it is arranged on the central axis of symmetry. The physical parameters of the coils are assumed in advance. The radius of the transmitter coil is $0.5 \mathrm{~m}$, the number of turns in the transmitter coil is 20 , and the current of the transmitter coil is set to $10 \mathrm{~A}$. The area of the receiver coil is $0.0001 \mathrm{~m}^{2}$, and the number of turns in the receiver coil is 20 . The parameters of the coils do 


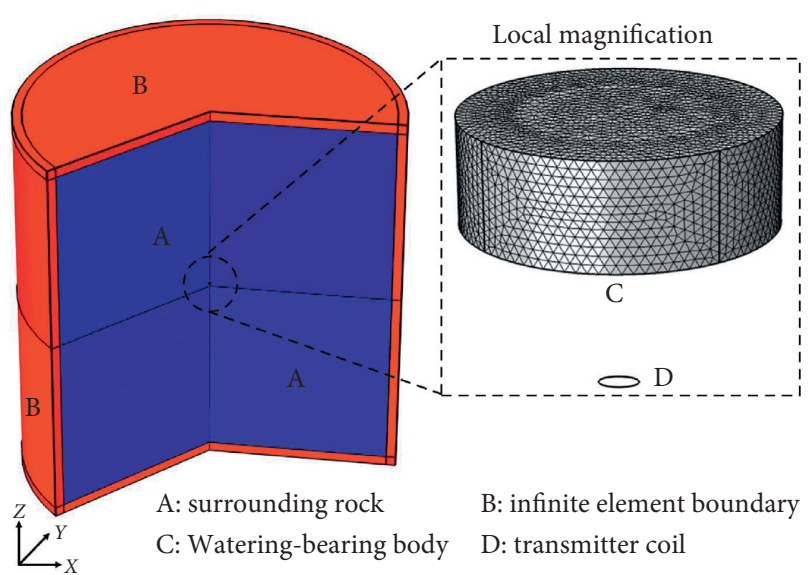

Figure 4: Layout of the finite element model.

not affect the overall shape of the curve of transient electromagnetic under the condition of ideal negative step current and uniform surrounding rock. The coils parameters mainly affect the early response of transient electromagnetic due to the inductive characteristics. Therefore, the parameters of the coils are unchanged in the qualitative analysis of this paper, and only the parameters of the VBB are focused.

According to formulas (4) and (5), the current is turned on at $0 \mathrm{~s}$, and the time-lapse transient electromagnetic response signal can be measured by the receiver coil when time is greater than $0 \mathrm{~s}$. To compare the transient electromagnetic response curve of the uniform full-space surrounding rock (UFSR) and WBB, the transient electromagnetic response of UFSR is also analyzed. The UFSR does not contain a WBB, and the resistivity of this region is equal to that of the surrounding rock in the simulation model. Due to the substantial dynamic contrast of the transient response, VBTEM time-lapse response signals are usually displayed on a log-log scale. Taking the induced voltage as the analysis object, the simulation results of the transient electromagnetic response curve of the UFSR and WBB are shown in Figure 5.

According to the simulation results of the transient electromagnetic response of UFSR, it can be concluded that the induced voltage response curve presents a linear relationship on the whole in the case of double logarithmic coordinates. However, it can be seen from Figure 5 that the variation in induced voltage in the presence of the WBB is obviously different from that in the UFSR model when the induction time is in the middle stage. At the same time, the induced voltage response curve of the WBB is consistent with the UFSR model when the induction time is in the early and late stages. In the middle stage, the induced voltage response curve is obviously characterized by three stages: uplift, peak (relief amplitude), and regression, as shown in Figure 5. Meanwhile, the effective time range level of the WBB is between $1.0 \times 10^{-7} \mathrm{~s}$ and $1.0 \times 10^{-4} \mathrm{~s}$ by comparing the response curves of UFSR and WWB; thus, the time requirement of VBTEM equipment at least microsecond level and even nanosecond level.

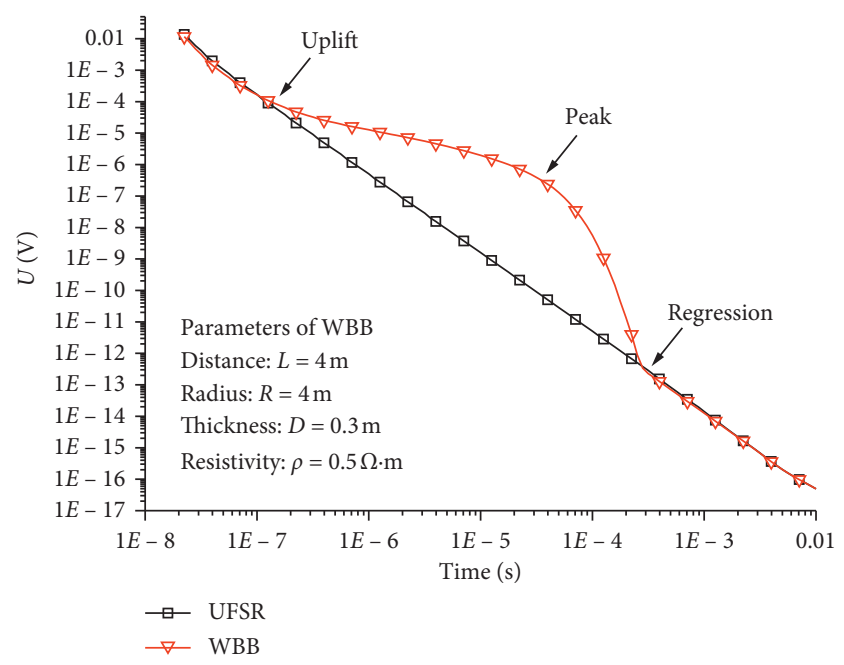

FIgURE 5: Transient electromagnetic response curve in the two models.

The transient electromagnetic signals of interest of the $\mathrm{WBB}$ are in the picosecond voltage range, and thus, the minimum signal value is too small to accurately measure. Drawing on other areas of detection technology, an effective transmitter-receiver moment-area needs to be redesigned, and heavy stacking and filtering are expected to increase the signal-to-noise ratio to an acceptable level $[10,17-19]$. Similarly, it is worth noting that the transient electromagnetic response curve of UFSR shows some fluctuation phenomena and decreases rapidly in the late stage of the transient electromagnetic response curve, which may be caused by model dimensions, mesh quality, and boundary conditions. However, a fluctuation phenomenon in the late stage exists in both the UFSR model and the WBB model, so this phenomenon does not affect the analysis of the transient electromagnetic response in the entire process.

2.4. Method of Characteristic Extraction. As shown by the result in Section 2.3, the transient electromagnetic response curves are obviously different in the presence of the WBB. Moreover, this difference is almost identical to the electromagnetic curves in other application fields $[9,14,15]$; thus, this conclusion provides a significant reference for us to apply VBTEM technology in operational tunnel detection. To quantitatively analyze the difference (i.e., the characteristics of the WBB), a method of characteristic extraction is proposed and the numerical characteristic parameters are extracted.

In the characteristic extraction method of the transient electromagnetic response, $\mathrm{Li}$ et al. [14] extracted the characteristic parameters of the curve through an empirical formula. However, this method has a certain randomness and thus requires many experiments to obtain the appropriate curve, and it also does not consider the influence of the background field on the response of the surrounding rock. The transient electromagnetic response of the 
background field of UFSR presents a linear change relation, which provides an important way for us to address the transient electromagnetic response of the WBB.

To compare the transient electromagnetic response of the UFSR and WBB, a mathematical method is used to divide the response of the UFSR by the response of the WBB, and this method can isolate abnormal data and highlight the change in the WBB. The mathematical treatment of the transient electromagnetic response of the WBB is shown in formula (6):

$$
I_{\mathrm{CP}}(t)=\frac{U_{\mathrm{WBB}}(t)}{U_{\mathrm{UFSR}}(t)},
$$

where $I_{\mathrm{CP}}(t)$ is the characteristic parameter formula after mathematical treatment, $U_{\mathrm{WBB}}(t)$ is the transient electromagnetic response formula of the $\mathrm{WBB}, U_{\mathrm{UFSR}}(t)$ is the transient electromagnetic response formula of the UFSR, and $t$ is the transient electromagnetic response time.

Taking the analysis object in Section 2.3 as an example, the result in double logarithmic coordinates after the mathematical treatment of formula (6) is shown in Figure 6. By comparing Figure 6 with Figure 5, we can find that the mathematical treatment method is essentially a kind of linear transformation, and this transformation illustrates that it will not cause data failure after changing data. Moreover, the results of the dimensionless response curve are independent of the parameters of the coil due to normalization.

According to the linear transformation of formula (6), seven numerical characteristic parameters can be extracted from Figure 6. These characteristic parameters mainly include the uplift point (UP), the maximum value (MV), the maximum point (MP), the regression point (RP), the left time span (LTS), the right time span (RTS), and the total time span (TTS). The UP indicates that the transient electromagnetic response curve of the WBB just exceeds that of the UFSR, which means that the transient electromagnetic response begins to focus on the WBB. The MV indicates that the characteristic parameter curve reaches its maximum value, which means that the response amplitude difference between WBB and UFSR reaches the maximum. The MP is expressed as the time to reach the maximum value. The RP illustrates that the transient electromagnetic response curve of the WBB just tends to that of the UFSR, which means that the transient electromagnetic response transforms from WBB to UFSR. The LTS represents the time when the characteristic parameter curve is in the increasing phase, and the RTS denotes the time when the characteristic parameter curve is in the decreasing phase. The TTS signifies the time from the uplift point to the regression point of the characteristic parameter curve, which is used to measure the total duration under the condition of the WBB. In addition, it should be noted that the LTS, the RTS, and the TTS are related to each other; that is, the third time span can be converted if the two other time spans are known.

Based on Li's research on the geological prediction of tunnels and our research objectives, the extraction process can be expressed by

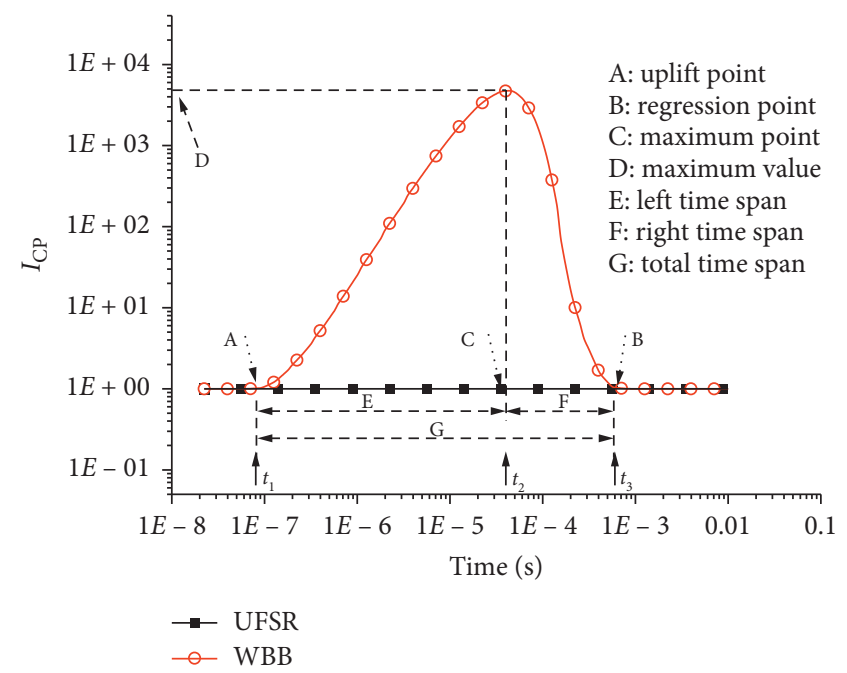

FIgURE 6: Characteristic parameters of the dimensionless response curve.

$$
\begin{aligned}
{[\mathrm{UP}, \mathrm{MV}, \mathrm{MP}, \mathrm{RP}, \mathrm{LTS}, \mathrm{RTS}, \mathrm{TTS}] } & =\operatorname{Ext}\left(I_{\mathrm{CP}}(t)\right) \\
& =\operatorname{Ext}\left(\frac{U_{\mathrm{WBB}}(t)}{U_{\mathrm{UFSR}}(t)}\right),
\end{aligned}
$$

where UP is the uplift point of the characteristic curve, $\mathrm{MV}$ is the maximum value of the characteristic curve, MP is the maximum point at the maximum value of the characteristic curve, RP is the regression point of the characteristic curve, LTS is the left time span of the characteristic curve, RTS is the right time span of the characteristic curve, TTS is the total time span of the characteristic curve, and Ext is the mathematical extraction method used to extract the seven numerical characteristic parameters.

The mathematical extraction equation of the characteristic parameters is shown by formula (8). Since the time span is very long, we use logarithmic time to represent the change in characteristic parameters, as shown in formula (8). Similarly, the logarithm of the maximum value is taken due to the huge change in its value. $t_{1}, t_{2}$, and $t_{3}$ represent the time of the UP, MP, and RP, respectively. $\alpha$ and $\beta$ are the judgment conditions for the uplift and regression of the response curve, their recommended values range from 1.05 to 1.25 , and their values are 1.15 in this paper.

$$
\begin{cases}\mathrm{UP}=\log \left(t_{1}\right), & E_{\mathrm{CP}}(t 1)>\alpha, \\ \mathrm{MV}=\log \left(E_{\mathrm{CP}}\left(t_{2}\right)\right), & \left(E_{\mathrm{CP}}\left(t_{2}\right)\right)^{\prime}=0, \\ \mathrm{MP}=\log \left(t_{2}\right), & \left(E_{\mathrm{CP}}\left(t_{2}\right)\right)^{\prime}=0, \\ \mathrm{RP}=\log \left(t_{3}\right), & E_{\mathrm{CP}}\left(t_{3}\right)<\beta, \\ \mathrm{LTS}=\log \left(t_{2}\right)-\log \left(t_{1}\right), & t_{2}>t_{1}, \\ \mathrm{RTS}=\log \left(t_{3}\right)-\log \left(t_{2}\right), & t_{3}>t_{2}, \\ \mathrm{TTS}=\log \left(t_{3}\right)-\log \left(t_{1}\right), & t_{3}>t_{1},\end{cases}
$$


TABLE 1: Parameter value of the sensitivity analysis in the numerical simulation.

\begin{tabular}{lcccc}
\hline Object & Distance $(\mathrm{m})$ & Radius $(\mathrm{m})$ & Thickness $(\mathrm{m})$ & Resistivity $(\Omega \cdot \mathrm{m})$ \\
\hline Distance & $2,4,6,8,10$ & 4 & 0.3 & 0.5 \\
Radius & 4 & $2,4,6,8,10$ & 0.3 & 0.5 \\
Thickness & 4 & 4 & $0.1,0.2,0.3,0.4,0.5$ & 0.5 \\
Resistivity & 4 & 4 & 0.3 & $0.1,0.2,0.5,1,5$ \\
\hline
\end{tabular}

where $t_{1}$ denotes the time of the UP, $\alpha$ is the condition for judging the UP of the characteristic parameter curve, $t_{2}$ represents the time to reach the $\mathrm{MV}, t_{3}$ indicates the time of the RP, $\beta$ is the condition for judging the RP of the characteristic parameter curve, and the notation log means taking the logarithm of time to the base 10 .

\section{Sensitivity Analysis of Transient Electromagnetic Parameters}

Based on Section 2.3, we find that the outstanding response characteristics of the WBB depend only on the physical parameters of the WBB itself when the parameters of the surrounding rock remain unchanged. In this section, four physical parameters that affect electromagnetic signals are selected in terms of distance, radius, thickness, and resistivity, and transient electromagnetic signal responses of the $\mathrm{WBB}$ are qualitatively investigated at different parameter levels. The parameter values of sensitivity analysis of transient electromagnetic parameters in the numerical simulation are shown in Table 1.

3.1. Influence Analysis of the Distance of the WBB. The transient electromagnetic response curve of the receiver coil at different distances of the WBB is depicted in Figure 7. When the WBB approaches the transmitter coil of the transient electromagnetic equipment, the relief amplitude of the transient electromagnetic response curve is obvious, the uplift time of the transient electromagnetic response curve is relatively early, and the regression time of the response curve tended to the transient electromagnetic response curve of UFSR is relatively late. Nevertheless, the relief amplitude of the response curve decreases significantly, and the duration of the relief amplitude of the transient electromagnetic response curve decreases obviously when the WBB is far from the transmitter coil.

Therefore, it can be concluded that the distance of the WBB greatly affects the uplift time and relief amplitude of the transient electromagnetic response curve. However, it is noteworthy that the distance of the $\mathrm{WBB}$ is not sensitive to the regression time of the transient electromagnetic response curve.

3.2. Influence Analysis of the Radius of the WBB. The transient electromagnetic response curve of the receiver coil at different radii of the WBB is illustrated in Figure 8 . When the radius of the $\mathrm{WBB}$ increases, the relief amplitude of the transient electromagnetic response curve is apparent, the

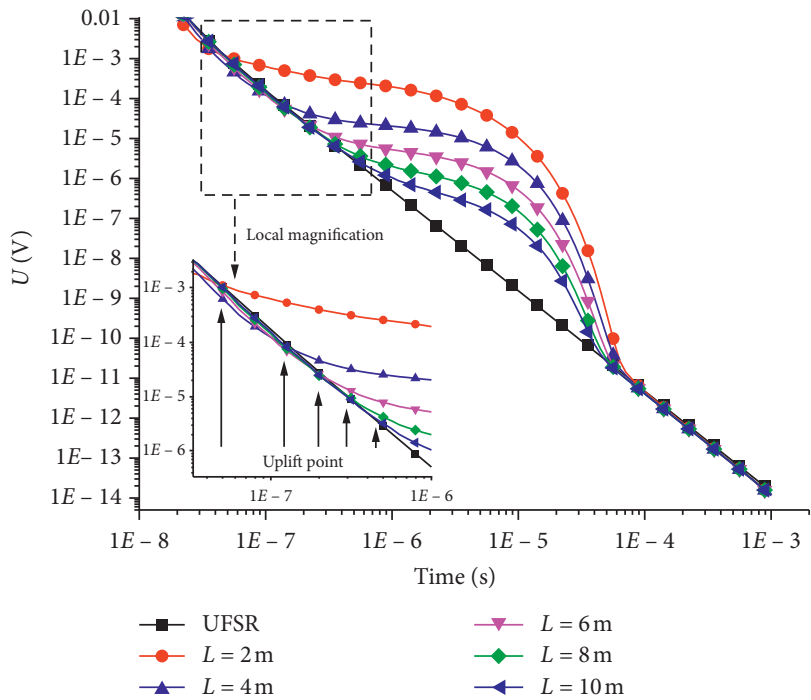

Figure 7: Response curve of the induced voltage of the WBB at different distances.

regression time of the transient electromagnetic response curve compared to the transient electromagnetic response curve of the UFSR is relatively late, and the uplift time of the transient electromagnetic response curve remains the same. Meanwhile, the relief amplitude of the transient electromagnetic response curve decreases significantly, and the regression time of the transient electromagnetic response curve is relatively early when the radius of the WBB decreases, so the duration of the relief amplitude of the transient electromagnetic response curve decreases obviously.

Based on above analysis, it can be concluded that the radius of the WBB clearly affects the relief amplitude and regression time of the transient electromagnetic response curve. Similarly, we find that the radius of the WBB does not affect the uplift time of the transient electromagnetic response curve.

3.3. Influence Analysis of the Thickness of the WBB. The transient electromagnetic response curve of the receiver coil at different thicknesses of the WBB is shown in Figure 9. When the thickness of the WBB increases, the relief amplitude of the transient electromagnetic response curve increases gradually but slowly, the regression time of the transient electromagnetic response curve compared to the transient electromagnetic response curve of the UFSR is relatively late, and the uplift time of the transient electromagnetic response curve remains the same. Meanwhile, the 


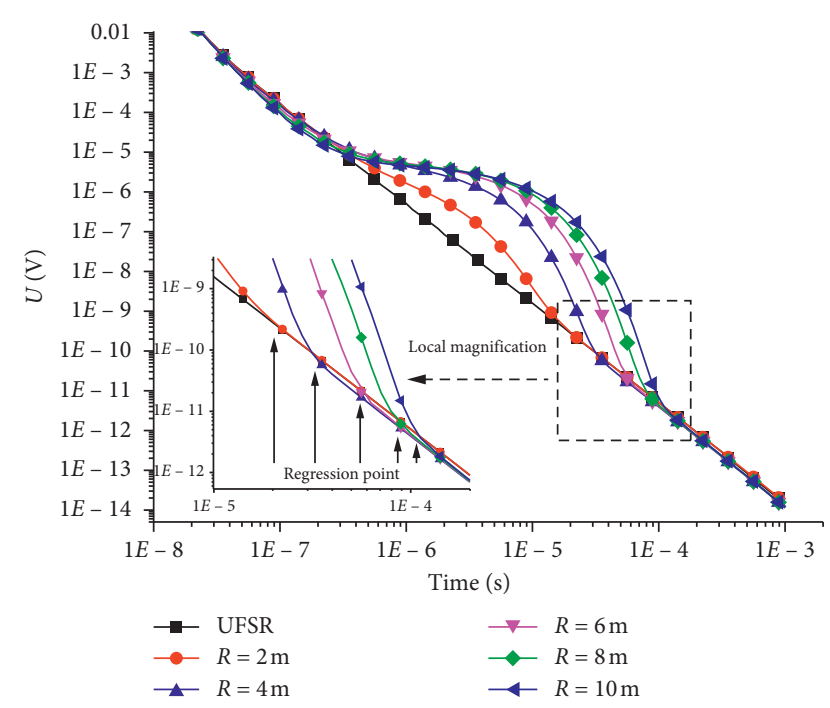

FIGURE 8: Response curve of the induced voltage of the WBB at different radii.

relief amplitude of the transient electromagnetic response curve does not decrease significantly, and the regression time of the transient electromagnetic response curve is relatively early when the thickness of the WBB gets smaller, so the duration of the relief amplitude of the transient electromagnetic response curve decreases slowly.

According to the parametric analysis of thickness, the thickness of the WBB affects the relief amplitude and regression time of the transient electromagnetic response curve to some extent. In the same way, we also know that the thickness of the WBB does not affect the uplift time of the transient electromagnetic response curve.

3.4. Influence Analysis of the Resistivity of the WBB. The transient electromagnetic response curve of the receiver coil at different resistivities of the WBB is presented in Figure 10. As shown, the relief amplitude of the transient electromagnetic response curve is significant and the regression time of transient electromagnetic response curve compared to the transient electromagnetic response curve of the UFSR is very late when the resistivity of the WBB decreases. The uplift time of the transient electromagnetic response curve remains essentially constant as the resistivity changes. When the resistivity of the WBB increases, meaning that the WBB has poor conductivity, the relief amplitude of the transient electromagnetic response curve decreases significantly, and the regression time of the transient electromagnetic response curve is very early, so the duration of the relief amplitude of the transient electromagnetic response curve decreases substantially. Therefore, the resistivity of the WBB clearly affects the relief amplitude and regression time of the transient electromagnetic response curve. Similarly, we find that the resistivity of the WBB does not affect the uplift time of the transient electromagnetic response curve.

According to the sensitivity analysis of the above four parameters, some qualitative conclusions can be drawn on

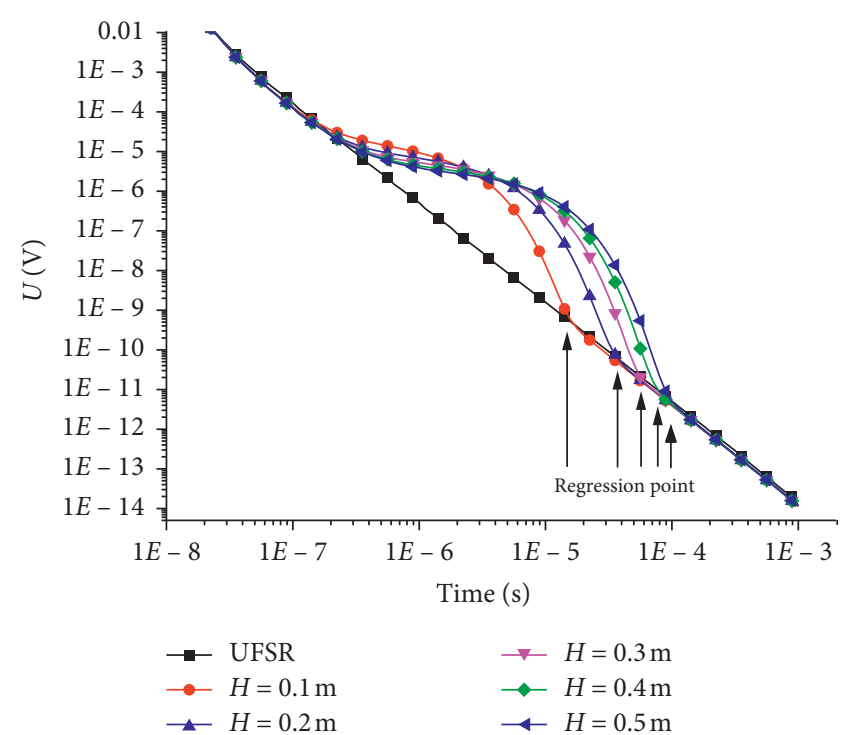

FIgURE 9: Response curve of the induced voltage of the WBB at different thicknesses.

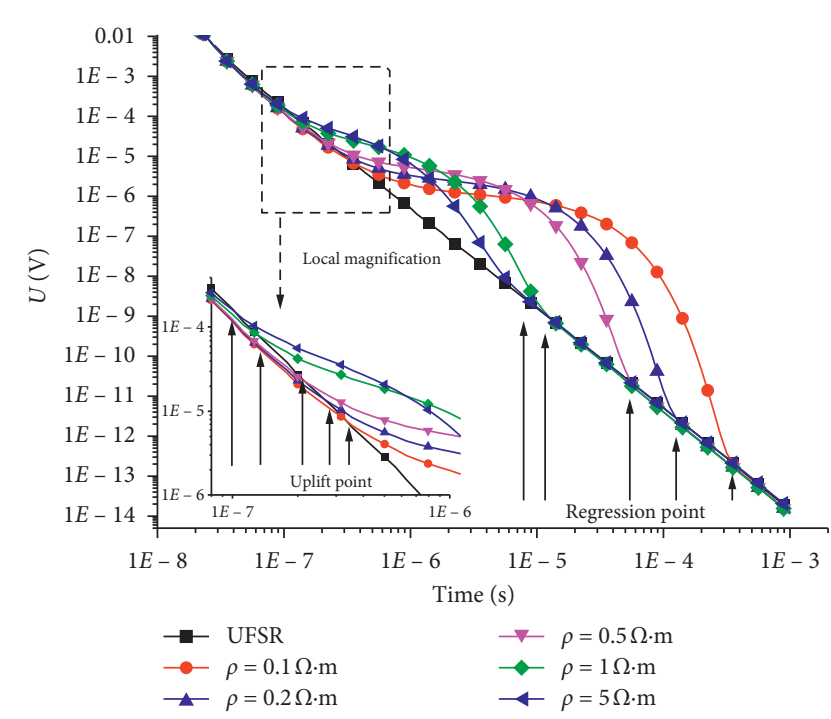

FIGURE 10: Response curve of the induced voltage of the WBB at different resistivities.

the electromagnetic outstanding response of the WBB. The influence of distance on the uplift time is much greater than that of other parameters, which means that the distance determines the uplift time. All four parameters influence the relief amplitude, indicating that the relief amplitude is a comprehensive manifestation of the physical parameters of the WBB. The regression time is affected by the radius, thickness, and resistivity, and the sensitivity order can be preliminarily estimated as resistivity, radius, and thickness.

\section{Extraction of Characteristic Parameter}

Based on Section 2.4 and Section 3, the results of the sensitivity parameter analysis can be analyzed to extract the 


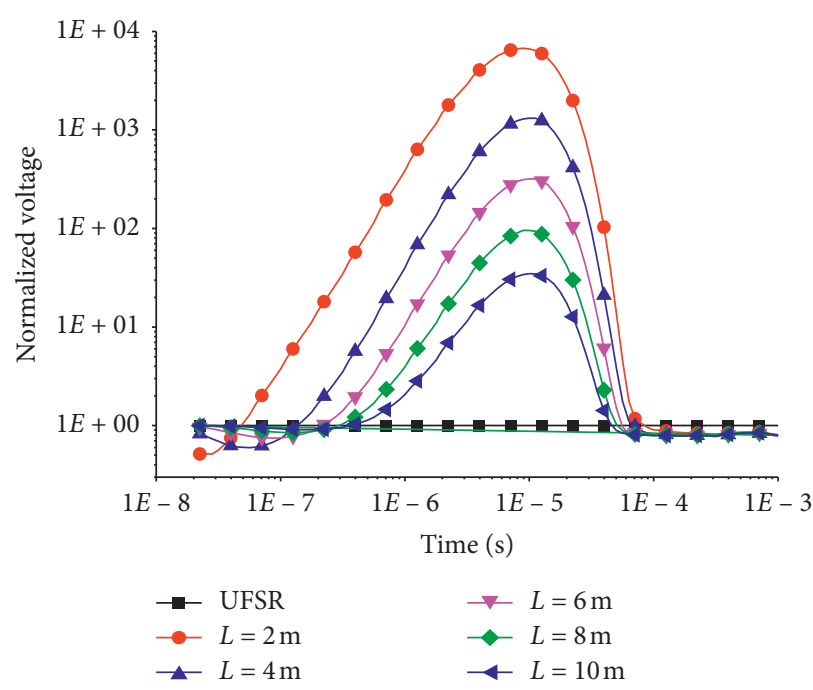

(a)

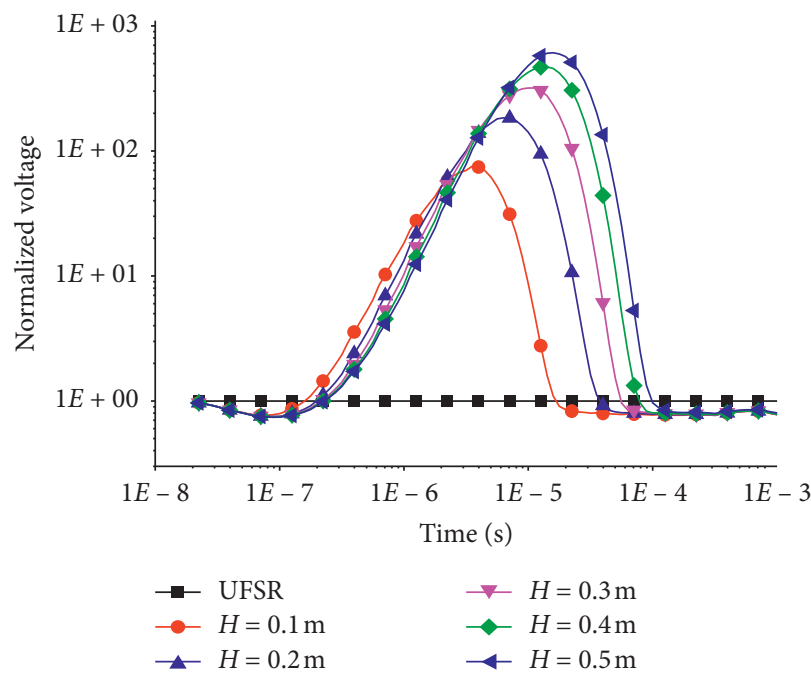

(c)

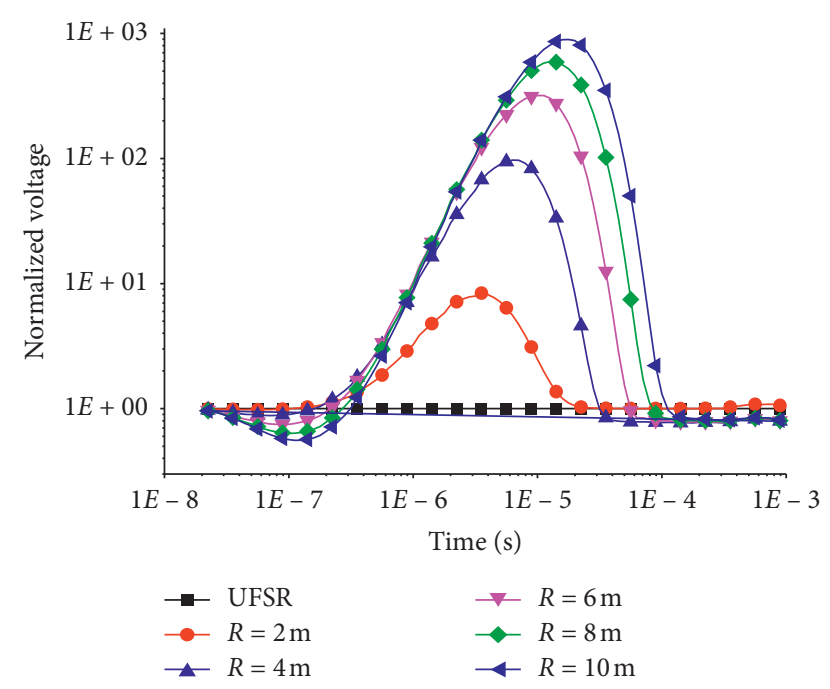

(b)

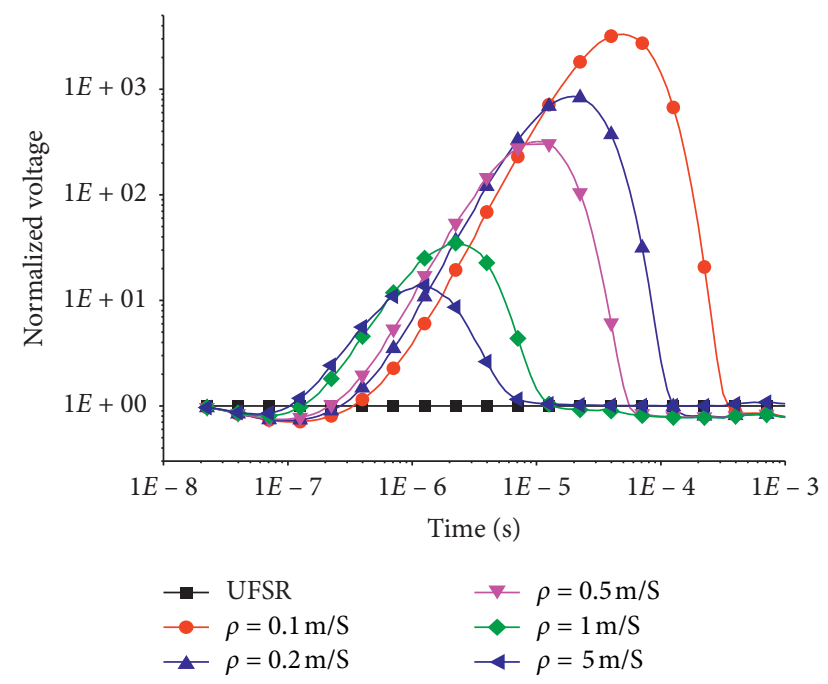

(d)

FIGURE 11: Dimensionless response curve of the WBB under different parameter conditions: (a) distance; (b) radius; (c) thickness; (d) resistivity.

numerical characteristic parameters of the WBB. The dimensionless response curve of the WBB under different parameter conditions is easily obtained by formula (6), as shown in Figure 11. The dimensionless curve is clearly differentiated, and the change rule of the WBB at different parameters is basically consistent with the results in Section 3.

The numerical characteristic parameters can be obtained by formulas (11) and (12), as shown in Table 2. The trends of the numerical characteristic parameters at different levels are shown in Figure 12 and 13. To facilitate the investigation of levels of different physical variables, the levels of different physical variables are expressed in alphabetical order, that is, $\mathrm{A}, \mathrm{B}, \mathrm{C}, \mathrm{D}$, and $\mathrm{E}$.

Figure 12 clearly shows that the radius and thickness have little influence on the UP at different levels, while the distance and resistivity have a significant influence on the UP. Furthermore, the UP increases significantly with increasing radius and decreases slowly with increasing resistivity. As shown in Figure 13, all four factors have a significant impact on the MV. The MV decreases gradually with increasing distance and resistivity in the logarithmic case. In contrary, the MV increases quickly with increasing radius and increases slowly with increasing thickness. As shown in Figure 12, the MP is not affected by the distance at different levels, and the other three factors affect the MP. In other words, the MP increases gently with increasing radius and thickness, and it decreases substantially with increasing resistivity. The RP is mainly affected by the radius, thickness, and resistivity, as shown in Figure 12, while the distance has almost no effect on the RP. Likewise, the RP increases substantially 
TABle 2: Numerical characteristic parameters of the dimensionless response curve at different parameters.

\begin{tabular}{|c|c|c|c|c|c|c|c|c|}
\hline Item & Level & $\begin{array}{l}\text { Uplift point } \\
\text { (UP) }\end{array}$ & $\begin{array}{c}\text { Regression point } \\
\text { (RP) }\end{array}$ & $\begin{array}{l}\text { Maximum point } \\
(\mathrm{MP})\end{array}$ & $\begin{array}{l}\text { Maximum value } \\
(\mathrm{MV})\end{array}$ & $\begin{array}{l}\text { Left time } \\
\text { span (LTS) }\end{array}$ & $\begin{array}{l}\text { Right time } \\
\text { span (RTS) }\end{array}$ & $\begin{array}{c}\text { Total time } \\
\text { span (TTS) }\end{array}$ \\
\hline$L=2 \mathrm{~m}$ & $\mathrm{~A}$ & -7.30 & -4.10 & -5.05 & 3.83 & 2.25 & 0.95 & 3.20 \\
\hline$L=4 \mathrm{~m}$ & B & -6.85 & -4.20 & -5.00 & 3.12 & 1.85 & 0.80 & 2.65 \\
\hline$L=6 \mathrm{~m}$ & $\mathrm{C}$ & -6.65 & -4.25 & -5.00 & 2.50 & 1.65 & 0.75 & 2.40 \\
\hline$L=8 \mathrm{~m}$ & $\mathrm{D}$ & -6.50 & -4.30 & -5.05 & 1.98 & 1.45 & 0.75 & 2.20 \\
\hline$L=10 \mathrm{~m}$ & $\mathrm{E}$ & -6.35 & -4.35 & -4.95 & 1.54 & 1.40 & 0.60 & 2.00 \\
\hline$R=2 \mathrm{~m}$ & A & -6.80 & -4.65 & -5.45 & 0.92 & 1.35 & 0.80 & 2.15 \\
\hline$R=4 \mathrm{~m}$ & B & -6.75 & -4.50 & -5.20 & 1.99 & 1.55 & 0.70 & 2.25 \\
\hline$R=6 \mathrm{~m}$ & $\mathrm{C}$ & -6.65 & -4.25 & -5.00 & 2.50 & 1.65 & 0.75 & 2.40 \\
\hline$R=8 \mathrm{~m}$ & $\mathrm{D}$ & -6.55 & -4.05 & -4.90 & 2.77 & 1.65 & 0.85 & 2.50 \\
\hline$R=10 \mathrm{~m}$ & $\mathrm{E}$ & -6.50 & -3.95 & -4.75 & 2.95 & 1.75 & 0.80 & 2.55 \\
\hline$H=0.1 \mathrm{~m}$ & A & -6.75 & -4.80 & -5.45 & 1.88 & 1.30 & 0.65 & 1.95 \\
\hline$H=0.2 \mathrm{~m}$ & B & -6.75 & -4.40 & -5.20 & 2.26 & 1.55 & 0.80 & 2.35 \\
\hline$H=0.3 \mathrm{~m}$ & $\mathrm{C}$ & -6.70 & -4.25 & -5.00 & 2.50 & 1.70 & 0.75 & 2.45 \\
\hline$H=0.4 \mathrm{~m}$ & $\mathrm{D}$ & -6.65 & -4.10 & -4.85 & 2.67 & 1.80 & 0.75 & 2.55 \\
\hline$H=0.5 \mathrm{~m}$ & $\mathrm{E}$ & -6.65 & -4.00 & -4.80 & 2.79 & 1.85 & 0.80 & 2.65 \\
\hline$\rho=0.1 \Omega \cdot \mathrm{m}$ & A & -6.45 & -3.45 & -4.35 & 3.52 & 2.10 & 0.90 & 3.00 \\
\hline$\rho=0.2 \Omega \cdot \mathrm{m}$ & B & -6.55 & -3.90 & -4.70 & 2.93 & 1.85 & 0.80 & 2.65 \\
\hline$\rho=0.5 \Omega \cdot \mathrm{m}$ & $\mathrm{C}$ & -6.65 & -4.25 & -5.00 & 2.50 & 1.65 & 0.75 & 2.40 \\
\hline$\rho=1.0 \Omega \cdot \mathrm{m}$ & $\mathrm{D}$ & -6.90 & -4.85 & -5.65 & 1.54 & 1.25 & 0.80 & 2.05 \\
\hline$\rho=5.0 \Omega \cdot \mathrm{m}$ & $\mathrm{E}$ & -7.00 & -4.95 & -5.95 & 1.15 & 1.05 & 1.00 & 2.05 \\
\hline
\end{tabular}

with increasing resistivity and decreases slowly with increasing radius and thickness level. Aimed at the short-distance TEM problem, the minimum time characteristic parameter is 7.3 when other conditions of the $\mathrm{WBB}$ remain unchanged; thus, the time requirement of the VBTEM equipment needs to reach the nanosecond level.

It can be concluded from Figure 13 that all four parameters substantially affect the time span characteristics, indicating that time span parameters are important characteristic parameters. Taking the TTS as an example, as shown in Table 2 and Figure 13, the TTS varies from 3.20 to 2.00 as the distance increases from $2 \mathrm{~m}$ to $10 \mathrm{~m}$, which means that the TTS decreases with increasing distance. The TTS changes from 2.15 to 2.55 as the radius increases from $2 \mathrm{~m}$ to $10 \mathrm{~m}$, which shows that the TTS increases with increasing radius. The TTS increases from 1.95 to 2.65 as the thickness increases from $0.1 \mathrm{~m}$ to $0.5 \mathrm{~m}$, which indicates that the TTS increases with increasing thickness. The TTS decreases from 3.00 to 2.05 as the resistivity increases from $0.1 \Omega \cdot \mathrm{m}$ to $5.0 \Omega \cdot \mathrm{m}$, meaning that the TTS decreases with increasing resistivity. As the physical parameters of the WBB change, the TTS of the transient electromagnetic signal also changes. In summary, this parameter-to-time relationship offers an intuitive way to interpret VBTEM signals from the surrounding rock.

\section{Technical Parameters of the VBTEM Equipment}

The technical parameters of the VBTEM equipment mainly include the first time gate, the last time gate, and the time acquisition interval. Based on seven characteristic parameters of the WBB, the main technical parameters of the VBTEM equipment are proposed.

Based on the quantitative analysis of characteristic parameters of uplift time and regression time, it can be found that the characteristic parameters of uplift time are mainly distributed in the range of -7.30 to -6.35 under different physical parameters, and the characteristic parameters of regression time are mainly distributed in the range of -4.95 to -3.45 . Obviously, the first time gate of the VBTEM equipment is much less than $1 \mu \mathrm{s}$, while the conventional transient electromagnetic shutdown time is generally in the range of several to dozens of microseconds. Therefore, the conventional transient electromagnetic equipment can only detect partial early-period signals, and most of the earlyperiod signals will be submerged in the equipment shutdown time. In terms of regression time, the last time gate of the VBTEM equipment is less than $1000 \mu \mathrm{s}$, and the middleperiod and late-period signals of the WBB can be measured by most conventional transient electromagnetic equipment, so the middle-period and late-period signals are not very strict on transient electromagnetic equipment. Therefore, it is recommended that the turn-off time of the VBTEM equipment should not exceed $0.05 \mu$ s to fully capture the early-period transient electromagnetic induction signals of the WBB.

Based on the quantitative analysis of characteristic parameters of time span, it can be found that the characteristic parameters of the TTS are mainly distributed in the range of 1.95 to 3.20 under different physical parameters. For conventional transient electromagnetic equipment, such as PROTEM 47 with a frequency of $237.5 \mathrm{~Hz}$, the starting value of the first time gate is $6.0 \mu \mathrm{s}$ and the starting value of the 20th time gate is $611.6 \mu \mathrm{s}$, so the logarithmic time span is 2.01. Obviously, in terms of time span, the equipment can 

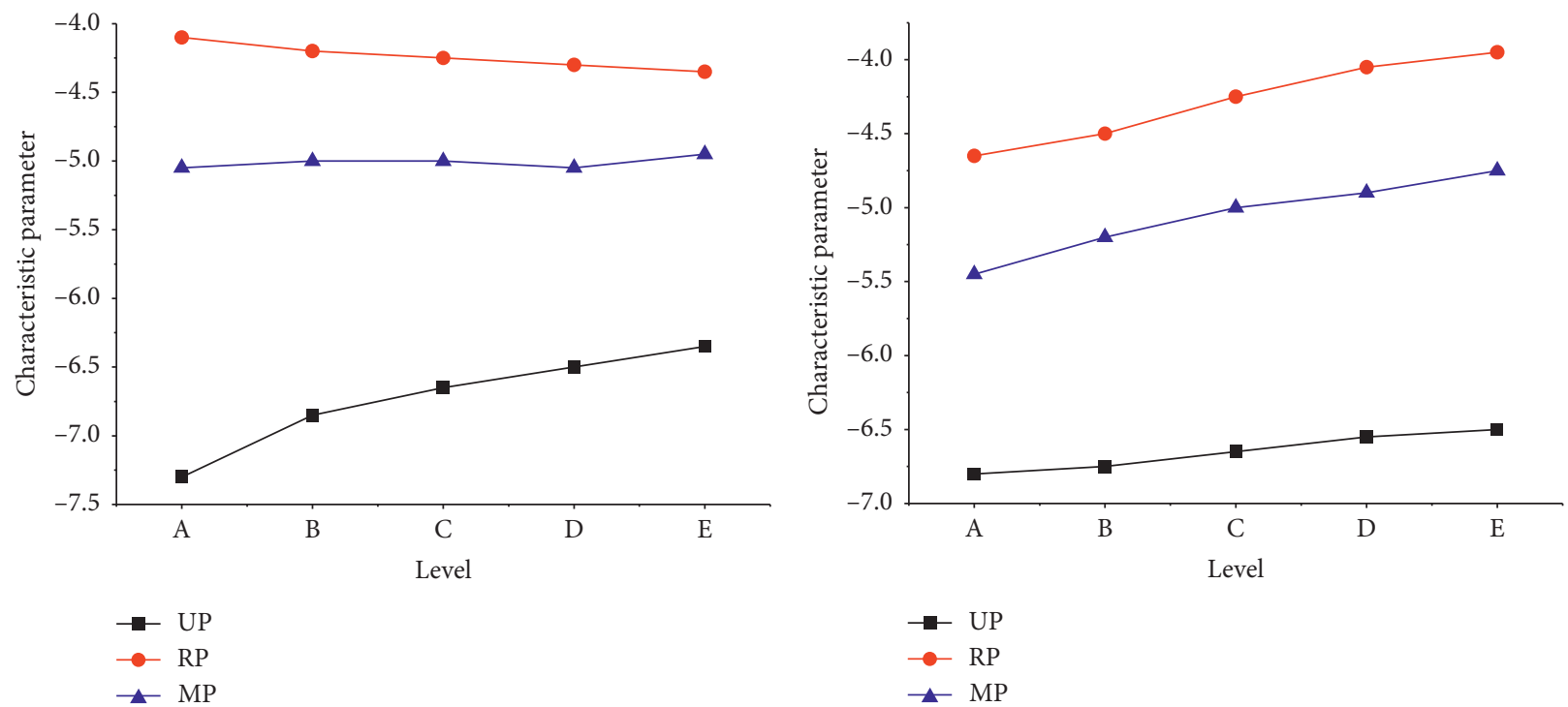

(a)

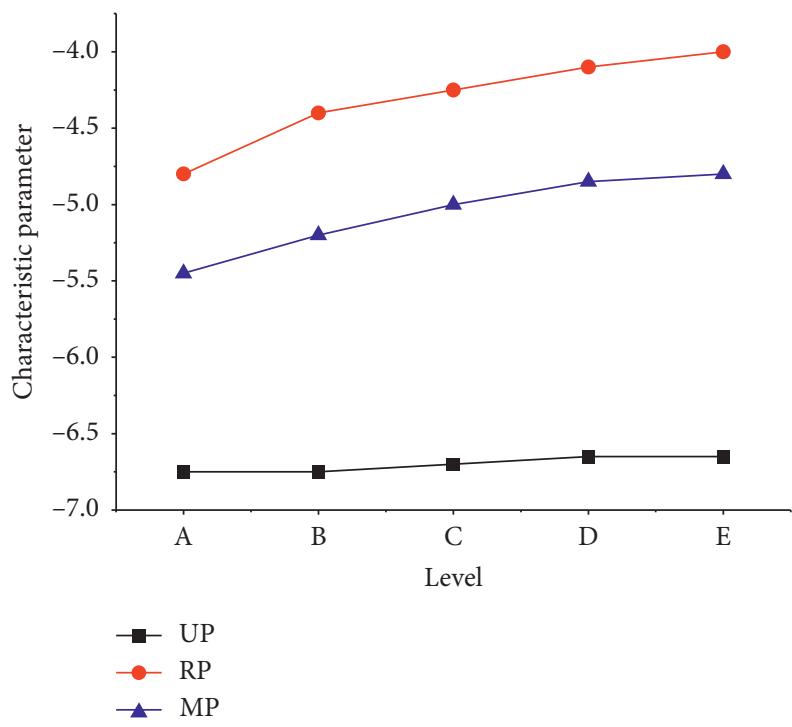

(b)

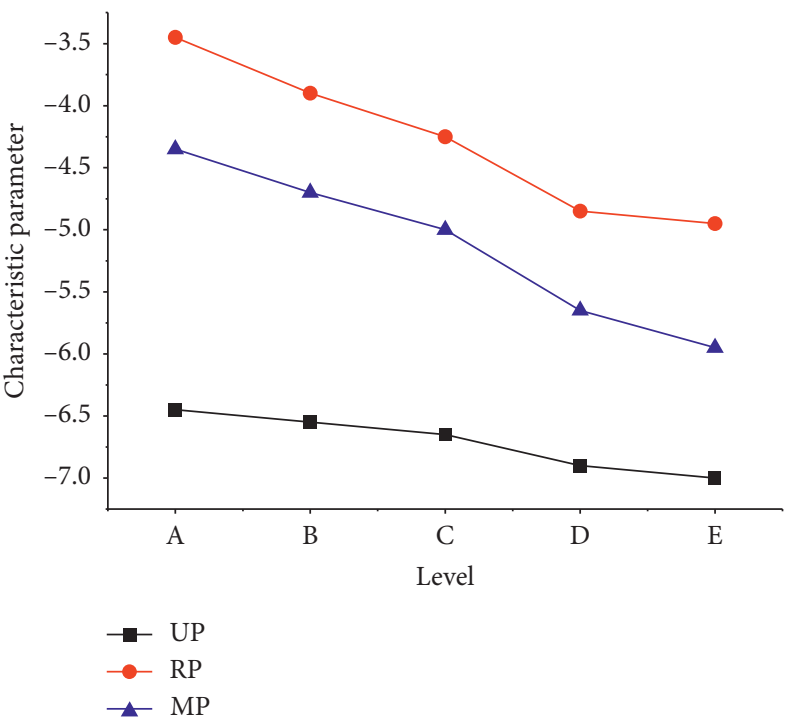

(c)

(d)

FIGURE 12: The first three numerical characteristic parameters of the WBB: (a) distance; (b) radius; (c) thickness; (d) resistivity.

only meet the electromagnetic response measurement of the WBB under a few conditions, and it is difficult to meet the electromagnetic response measurement of the WBB in most conditions. In order to fully obtain the full-period transient electromagnetic induction signals of the $\mathrm{WBB}$, the time span of the VBTEM equipment should be at least 1.95 in the small-scale or long-distance of the WBB, and the time span must be at least 3.20 in the large-scale or short-distance WBB.

It should be noted that the above time span is only for the prominent response of the $\mathrm{WBB}$, and it does not consider the part of the response that is consistent with that of UFSR. Actually, the vehicle-borne transient electromagnetic equipment also needs to consider the response data of surrounding rock, so it is suggested that the time span of the VBTEM equipment can reach four time spans to better realize the comparative analysis of field detection data and response data of surrounding rock.

\section{Discussion}

The numerical characteristic parameter of VBTEM is mainly obtained via the numerical simulation. In the follow-up investigation, these results need to be verified by both theoretical and experimental methods. The current in the transmitter coil cannot be turned off suddenly, and there is a transient process in the coil $[9,16,20]$. The transmitterreceiver coil design of VBTEM is different from the coil design in other electromagnetic fields, and it also need to be specially designed. If the key problems mentioned above can be solved, the method of VBTEM can be applied to the rapid detection of lining disease in operating tunnels. The 


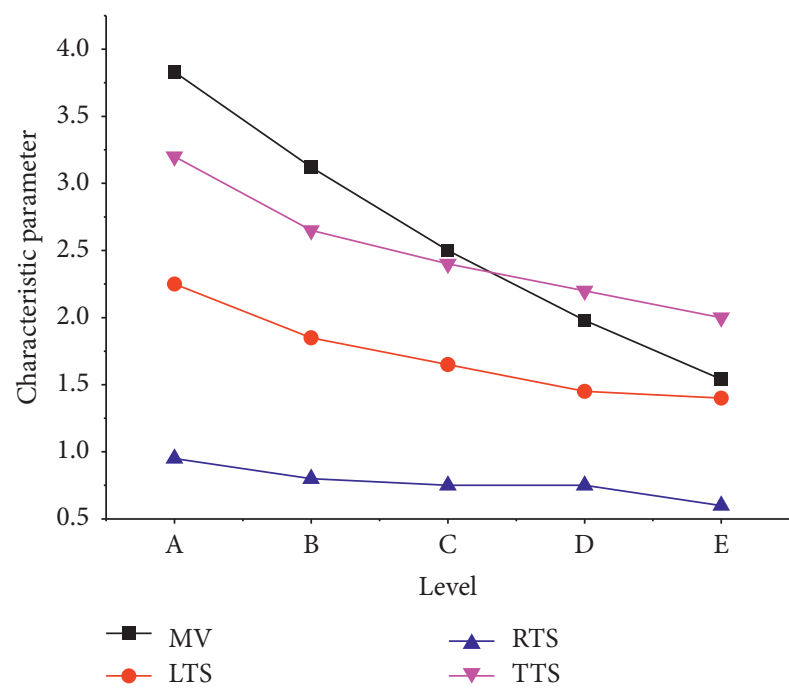

(a)

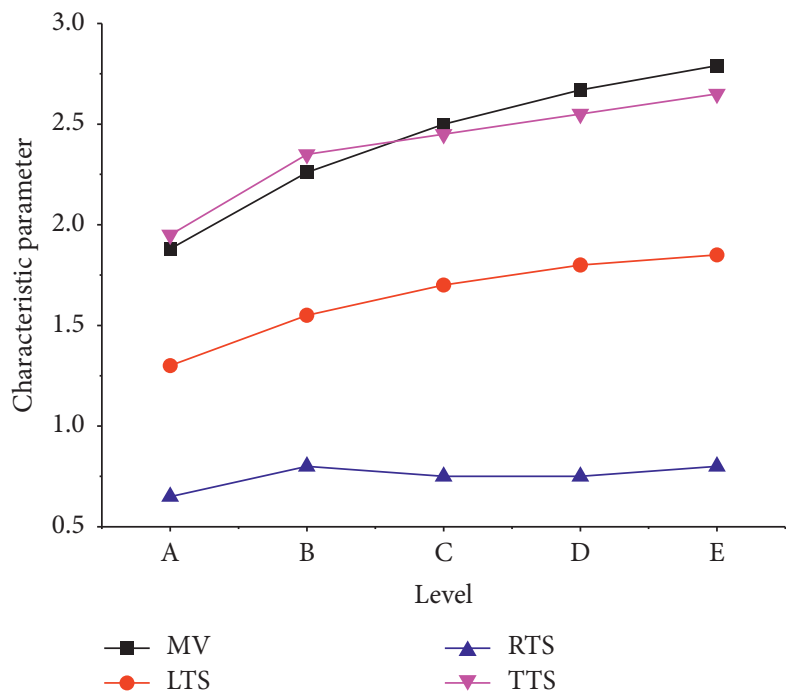

(c)

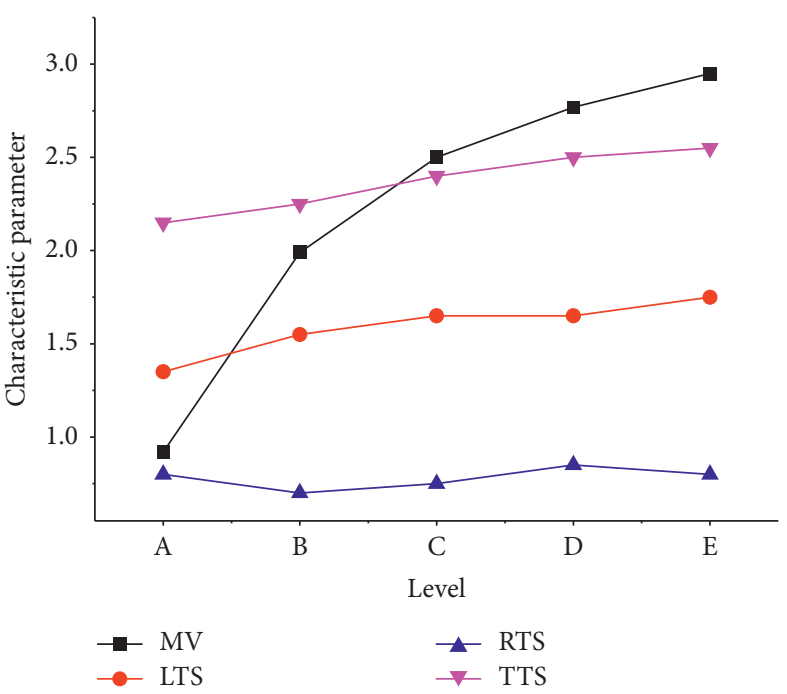

(b)

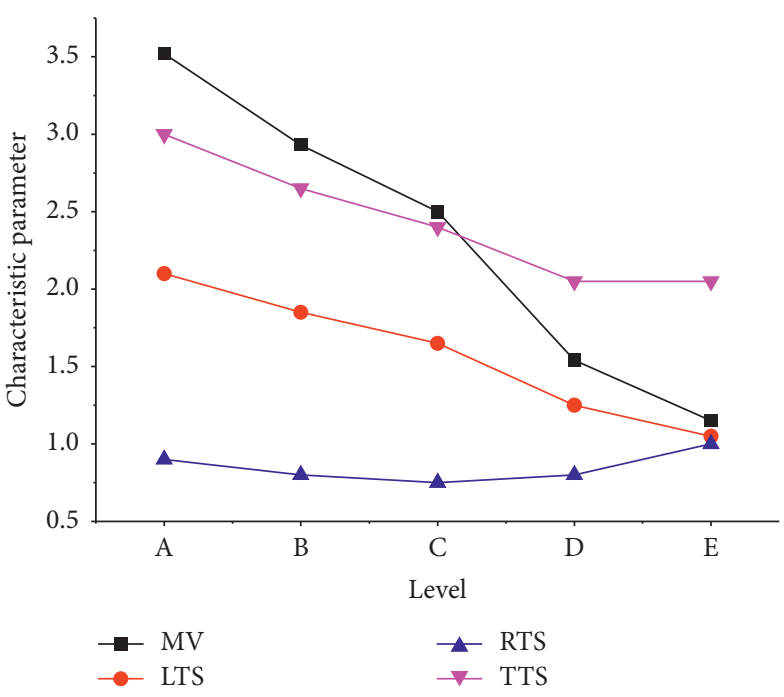

(d)

FIgURE 13: The last four numerical characteristic parameters of the WBB: (a) distance; (b) radius; (c) thickness; (d) resistivity.

detection result of VBTEM can provide a detailed location and distribution interval of the WBB for the maintenance of lining disease in operational tunnels.

\section{Conclusion}

In this paper, the transient electromagnetic response characteristics of the WBB behind tunnel linings is investigated in terms of the response curves, time range, and scale of water-bearing body based on the parametric sensitivity analysis. Analysis of the results of the numerical simulation and mathematical extraction equation convince us of the following. (1) There is a significant difference between the transient electromagnetic response of the WBB and UFSR, and this difference provides the possibility to detect the lining disease in operating tunnels. (2) According to the idea of normalization, quantification of dimensionless response curves is proposed, and seven numerical characteristic parameters are extracted. This parameter-to-parameter relationship offers an effective and intuitive way to interpret VBTEM signals from the surrounding rock. (3) Based on the parametric sensitivity analysis, the technical parameters requirements of the VBTEM equipment are proposed. The first time gate of the VBTEM equipment need to not exceed $0.05 \mu$ s for short-distance TEM problem, and the time span of the VBTEM equipment should reach four time spans to fully capture the electromagnetic response signal. Hence, the research results can provide valuable technical references for the application of VBTEM.

\section{Data Availability}

The data used to support the findings of this study are available from the corresponding author upon request. 


\section{Conflicts of Interest}

The authors declare that there are no conflicts of interest regarding the publication of this paper.

\section{Acknowledgments}

The National Natural Science Foundation of China (grant nos. 51478395 and 5197080874) are acknowledged for their financial support. The authors would like to thank Ms. Wenjing Lu from the Affiliated Middle School of Jiangsu Normal University, China, for linguistic assistance during the preparation of this manuscript.

\section{References}

[1] T. Asakura and Y. Kojima, "Tunnel maintenance in Japan," Tunnelling and Underground Space Technology, vol. 18, no. 2, pp. 161-169, 2003.

[2] M. A. Meguid and H. K. Dang, "The effect of erosion voids on existing tunnel linings," Tunnelling and Underground Space Technology, vol. 24, no. 3, pp. 278-286, 2009.

[3] M. Lei, L. Peng, C. Shi, and S. Wang, "Experimental study on the damage mechanism of tunnel structure suffering from sulfate attack," Tunnelling and Underground Space Technology, vol. 36, no. 6, pp. 5-13, 2013.

[4] S. C. Li, Z. Q. Zhou, Z. H. Ye, L. P. Li, Q. Q. Zhang, and Z. H. Xu, "Comprehensive geophysical prediction and treatment measures of karst caves in deep buried tunnel," Journal of Applied Geophysics, vol. 116, no. 5, pp. 247-257, 2015.

[5] J. Sun, "Durability problems of lining structures for Xiamen Xiang'an subsea tunnel in China," Journal of Rock Mechanics and Geotechnical Engineering, vol. 3, no. 4, pp. 289-301, 2011.

[6] T.-T. Wang and C.-H. Lee, "Life-cycle design considerations for hydraulic tunnels: lessons learned from inspection and maintenance cases," Journal of Performance of Constructed Facilities, vol. 27, no. 6, pp. 796-806, 2013.

[7] D. V. Fitterman and M. T. Stewart, "Transient electromagnetic sounding for groundwater," Geophysics, vol. 51, no. 4, pp. 995-1005, 1986.

[8] A. Hordt, P. Andrieux, F. Neubauer, H. Ruter, and K. Vozoff, "A first attempt at monitoring underground gas storage by means of time-lapse multichannel transient electromagnetics," Geophysical Prospecting, vol. 48, no. 3, pp. 489-509, 2000.

[9] J. E. Danielsen, E. Auken, F. Jørgensen, V. Søndergaard, and K. I. Sørensen, "The application of the transient electromagnetic method in hydrogeophysical surveys," Journal of Applied Geophysics, vol. 53, no. 4, pp. 181-198, 2003.

[10] D. Tan, "Research on theory and application for advance prediction of water by whole space transient electromagnetism," Doctor's thesis, Southwest Jiaotong University, Chengdu, China, 2009.

[11] I. N. Yeltsov, G. V. Nesterova, and A. A. Kashevarov, "Petrophysical interpretation of time-lapse electromagnetic sounding in wells," Russian Geology and Geophysics, vol. 52, no. 6, pp. 668-675, 2011.

[12] S. Li, B. Liu, X. Xu et al., "An overview of ahead geological prospecting in tunneling," Tunnelling and Underground Space Technology, vol. 63, no. 3, pp. 69-94, 2017.

[13] L. Bin, L. Zhengyu, L. Shucai et al., "Comprehensive surface geophysical investigation of karst caves ahead of the tunnel face: a case study in the Xiaoheyan section of the water supply project from Songhua River, Jilin, China," Journal of Applied Geophysics, vol. 144, no. 9, pp. 37-49, 2017.

[14] Y. Li, T. Yue, B. Lei, Z. Li, and W. Qian, "An iterative inversion method using transient electromagnetic data to predict water-filled caves during the excavation of a tunnel," Geophysics, vol. 84, no. 2, pp. 1-16, 2019.

[15] S. Li, H. Sun, X. Lu, and X. Li, "Three-dimensional modeling of transient electromagnetic responses of water-bearing structures in front of a tunnel face," Journal of Environmental \& Engineering Geophysics, vol. 19, no. 1, pp. 13-32, 2014.

[16] H. Sun, X. Li, S. Li et al., "Three-dimensional FDTD modeling of TEM excited by a loop source considering ramp time," Chinese Journal of Geophysics, vol. 56, no. 3, pp. 1049-1064, 2013.

[17] R. S. Smith and A. P. Annan, "Using an induction coil sensor to indirectly measure the B-field response in the bandwidth of the transient electromagnetic method," Geophysics, vol. 65, no. 5, pp. 1487-1494, 2000.

[18] G.-q. Xue, C.-y. Bai, S. Yan, S. Greenhalgh, M.-f. Li, and N.-n. Zhou, "Deep sounding TEM investigation method based on a modified fixed central-loop system," Journal of Applied Geophysics, vol. 76, no. 1, pp. 23-32, 2012.

[19] T. Qi, D. Tan, and Z. Wu, "Physical simulation of the transient electromagnetic response throughout a whole tunnel," Modern Tunnelling Technology, vol. 50, no. 1, pp. 53-59, 2013.

[20] N. O. Kozhevnikov, "Current turn-off in an ungrounded horizontal loop: experiment and theory," Russian Geology and Geophysics, vol. 57, no. 3, pp. 498-505, 2016. 